\title{
The Performance Optimization of Energy-Efficient Scheduling Algorithm for Cluster-based Wireless Sensor Networks
}

\author{
Hsing-Wen Wang ${ }^{1}$ and Pin-Jui Chen ${ }^{2}$ \\ ${ }^{1}$ Department of Business Administration, \\ National Changhua University of Education \\ ${ }^{2}$ Department of Communication Engineering, \\ National Central University \\ Email: shinwen@cc.ncue.edu.tw \\ jeffery2936@hotmail.com
}

\begin{abstract}
The use of wireless sensor networks is very broad, and its application can be extended to a number of areas. In general, after the wireless sensor networks have been set up, there is no maintenance needed. The life cycle of the network will be limited only by the battery power supply of the sensors. Once the entire network energy consumption causes loss of balance in the system, the energy hole problem will occur, and even cause paralysis of the part of the system. In the cluster architecture, the burden of the cluster heads is very large, so it is bound to become the point with the largest energy consumption. Therefore, we will focus on creating an energy-saving method for the cluster heads. For the new schedule algorithm in our approach, we used the "polling" approach, so that the cluster heads can have effective and absolutely correct data reception and transmission. In addition, we also introduced the "sleeping" mechanism to ensure that the cluster head can save power through the most effective methods of data reception. Through two main mechanisms, not only will the overall operation function more efficiently, but also achieve the purpose of substantially prolonging the life expectancy of the whole network.
\end{abstract}

Keywords-Scheduling Policy; power saving; wireless sensor networks; clustering

\section{INTRODUCTION}

Wireless sensor network is set by multiple sensing devices in the target environment, and achieve the purpose of monitoring the environment by collecting the environmental data through the wireless network [1] [2]. There are a huge amount of sensor nodes sense data and sent to the data collection point, therefore, many protocol for data transmission were been developed. One of them is to transmit information by node relaying, which is called the multi-hop transmission [3]. In the multiple-hop transmission method, the nodes farther away from data collection point will send data to the nodes closer to the base station first; this method will avoid a large amount of energyconsuming because of the transmission from distant distance [4].

However, the nodes closer to the base station need to relay the data from the other nodes very frequently, therefore the energy consumption will be particularly high. When the loss of these nodes happens, the transmission distance of other nodes will be increased, and the energy consumption will also be increased; these will cause a failure of data transmission. As a result, it will lead to the partial sensing node to lose effectiveness. Even more, an energy hole problem will be generated. As the following diagram shown in Fig. 1, the energy consumption of the overall system will be extremely uneven, and leading to the shortening of life cycle [5].

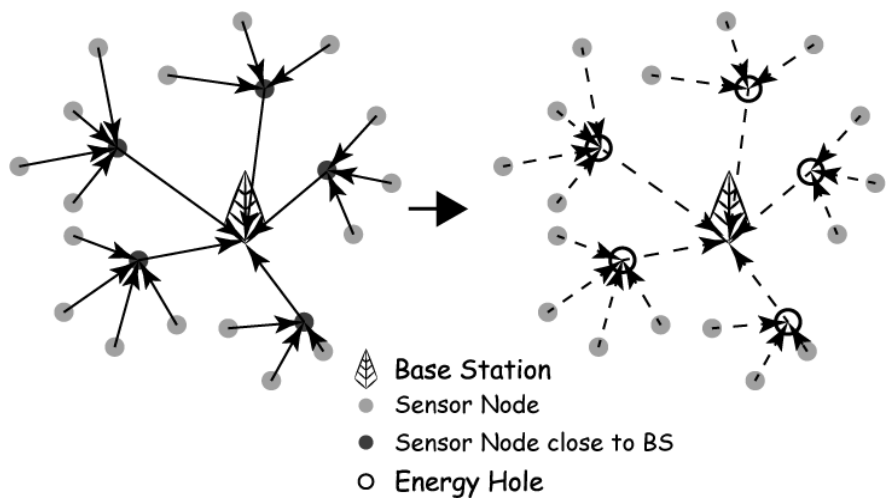

Fig. 1. Energy hole problem.

The energy-hole problem cannot be completely avoided; we can only attempt to reduce the occurrence of this problem. Thus, the energy consumption of each node must be balanced in order to help avoid the energy hole problem caused by the loss of partial sensing nodes. That is, to extend the life cycle of the system by balancing the energy distribution [6] [7].

The cluster infrastructure concept divides the geographical area of sensor network into more than one cluster, then each cluster has one data collector as a cluster head [8], the members of the cluster just send data to the cluster head, and the cluster head will passed the data collected from the cluster to the base station [9] [10].

In cluster's infrastructure architecture, cluster heads usually become the point that has the heaviest energy load, and were easily found to cause the energy hole. In this paper, we take a particular scheduling method to make cluster head achieve the optimal power saving schedule under the premise that it satisfies the time tolerance. 
The next topic is organized as follows: II mainly to explain the network model and architecture defined by this paper, III Is telling about the operation, IV is the mathematical proof part, $\mathrm{V}$ for the computer simulation results, and finally, VI is the conclusion of this paper.

\section{NeTWORK MODEL AND ARCHITECTURE}

In this section, we first introduce the general basic wireless sensor network, and then define the network environment we used.

\section{A. Network Model}

Wireless sensor network is mainly constituted by the data collection center (Sink Node) and some sensor nodes. Sensor nodes can be homogeneous, which means that each node has the same ability (electricity, computing ability and etc.), but also can be heterogeneous, when the sensor network is constituted by the nodes with different abilities.

Transmission of wireless sensor networks can be divided into two types: direct transmission and multi-hop transmission. Mostly by using the multi-hop transmission to transmit data by nodes relaying to the data collection point, data is transmitted in several steps in order to increase the effective sensing scope.

\section{B. Network Architecture}

We use cluster-based network architecture, using the homogeneous sensor nodes, but divide them into two classes: a cluster head and several cluster nodes. Cluster is a circle; the cluster head as the center, and the distance $\mathrm{R}$ is the radius of the circle. The deployment of the nodes is a topology formed by taking random uniform distribution.

Cluster nodes are responsible for sensing data from the environment, and passing it to the cluster head. The cluster head, responsible for compressing the received data, passes to the cluster head closer to the sink node, and finally the sink node pass to users.

\section{PROPOSED SCHEME}

The proposed scheme is divided into two stages. The first stage is the Initial Phase. In this stage, the formation of the cluster will be determined. And the second is the Running Phase, that is, the beginning of data transmission.

\section{A. Initial Phase}

\section{1) Cluster Set Up}

Function Cluster_Initializing

WHILE

Head broadcasts the Ini-msg.

Head receives the Join-req from node $i$.

WHILE not timeout DO

IF node $i$ satisfy $\delta^{*}<\delta$ and $\tau<\delta *$ THEN

Head allow node $i$ to join in this cluster.

Create a token buffer for the node.

END IF

END WHILE

END WHILE
The cluster head must tell other nodes in the network that it is the cluster head. Cluster head broadcasts an Ini-msg (initial message) to all nodes in the cluster at first. The Ini-msg contains an ID of the cluster head and some environmental parameters, such as sample rate, channel rate, etc. After each non-clusterhead node receives the Ini-msg, it will transmit a Join-req (join request) message to ask for joining the cluster by using CSMA/CA protocol [11]. After the cluster head receives the Join-req message, it will based on the relevant environmental parameters to decide whether to accept this request (the conditions and their proof see section 2 of A).
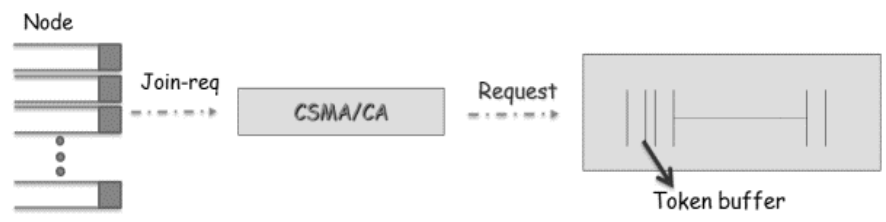

Fig. 2. Cluster set up.

Once the non-cluster-head node has been joined, the cluster head will create a corresponding token buffer [12] as Fig. 2 shows. The cluster head will keep receiving Join-req messages until it runs out of time or reaches the upper bound of node numbers. After that, the cluster head will send a broadcast message that contains a list of members that has successfully joined to the cluster to inform that it is the end of the initialization, then the cluster head will enter the stable state.

\section{2) Join A Cluster}

The cluster head must ensure that the cluster node had been added can satisfy a certain time to meet its tolerance time. Only when $\delta^{*}<\delta, \tau<\delta^{*}$ and $\tau=(\mathrm{i}-1) \times \mathrm{T}_{\mathrm{p}}$, the cluster head will accept the request [13]. So we have to prove that $\tau=(i-1) \times$ $\mathrm{T}_{\mathrm{p}}<\delta^{*}$.

Proof: When $\delta^{*}<\delta, \tau<\delta^{*}$ and $\tau=(i-1) \times$ $T_{p}$ are true, $\tau=(i-1) \times T_{p}<\delta^{*}$ will be true.

First, we assume some variable as below:

$i$ : Number of nodes in the cluster.

$T_{p}$ : Time for a round time of node processing.

$\delta^{*}=i \cdot T_{p}$

$\delta$ : The delay time that a cluster head could tolerate.

$\tau$ : Maximun waiting time.

Step 1 .

When $i=1$, it can establishes the induction basis.

i.e. $\tau=(1-1) \times \mathrm{T}_{\mathrm{p}}=0 \times \mathrm{T}_{\mathrm{p}}=0<\delta^{*}$

Step 2.

Suppose our induction hypotheses hold up to $i=k$.

i.e. $\tau=(\mathrm{k}-1) \times \mathrm{T}_{\mathrm{p}}<\delta^{*}$

Step 3.

$$
\begin{aligned}
& \text { When } \mathrm{i}=\mathrm{k}+1, \tau=(\mathrm{k}+1-1) \times \mathrm{T}_{\mathrm{p}}=\mathrm{k} \times \mathrm{T}_{\mathrm{p}} \\
& \because \frac{\delta^{*}}{\mathrm{~T}_{\mathrm{p}}}<\frac{\delta}{\mathrm{T}_{\mathrm{p}}} \therefore \mathrm{k}+1<\frac{\delta}{\mathrm{T}_{\mathrm{p}}} \\
& \because \mathrm{k} \times \mathrm{T}_{\mathrm{p}}+\mathrm{T}_{\mathrm{p}}<\delta^{*} \quad \therefore \mathrm{k} \times \mathrm{T}_{\mathrm{p}}<\delta^{*}
\end{aligned}
$$


This shows that $\delta^{*}<\delta, \tau<\delta$ and $\tau=(N-1) \times T_{p}$, $=(N-1) \times T_{p}<\delta$, for all $N$. Based on the principle of induction, the statement of the theorem follows Q.E.D.

\section{B. Running Phase}

When the system are in this stage, it can be divided into four mechanisms: (1)Token Buffer Mechanism, (2)Detection Mechanism, (3)Polling Mechanism, (4)Node Processing, four mechanisms work as follows:

\section{1) Token Buffer Mechanism}

The cluster head establishes a token buffer; the token buffer products token over sample rate, to let it know that it should receive the data from the corresponding node.

\section{2) Detection Mechanism}

\section{Function Polling_List_Creating}

\section{WHILE}

Head scans all nodes in the cluster.

IF there is a token has been scanned THEN Add the node ID to the Polling List. Pointer moves back to the first buffer.

ELSE

Scan again from the first buffer to the last.

\section{END IF}

\section{END WHILE}

In Fig. 3, the cluster head scans every token buffer. Once finding the Token, it will add node ID into the Polling List and start scanning again from the first token buffer.
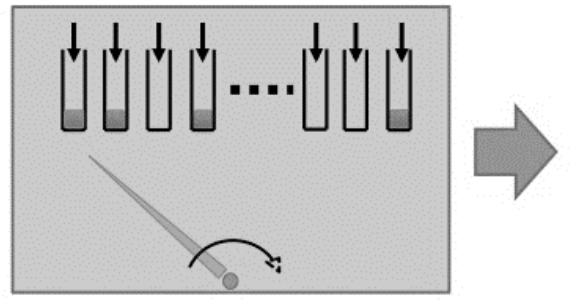

\begin{tabular}{|c|c|}
\hline Node ID & State \\
\hline 1 & Polling \\
\hline 19 & Waiting \\
\hline 12 & Waiting \\
\hline$\ldots$ & $\ldots$ \\
\hline$\ldots$ & $\ldots$ \\
\hline & \\
\hline
\end{tabular}

Fig. 3. Detection mechanism.

\section{3) Polling Mechanism}

\footnotetext{
Function Poll_Sending

WHILE

IF Polling List is not empty THEN

Head turns to active mode.

Send the Polling-pkt to node $i$.

ELSE

Head turns to sleeping mode.

END IF

\section{END WHILE}

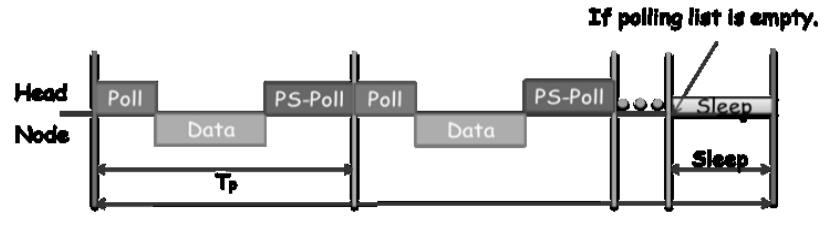

Fig. 4. Polling mechanism.

As shown in Fig. 4, if Polling List is empty, the cluster head will keep in sleeping mode. If Polling List is not empty, the cluster head will switch to active mode. Then it sent a Pollingpkt to the corresponding cluster node, the node will know that it should deliver the data to the cluster head. When the cluster node received the Polling-pkt, it started to deliver the data until the cluster head reply a PS-Poll (power saving poll) packet [14] and removes the node ID from the Polling List. After the completion of action system will restart Polling Mechanism.

\section{4) Node Processing}

The cluster node keeps in sleeping mode and sensing data. When the node sensed a data, the device will switch into active mode and receiving the Polling-pkt from the cluster head. Once the cluster node receives $P S-P$ oll packet, the node will switch to sleeping mode again, Fig. 5 shown as below.

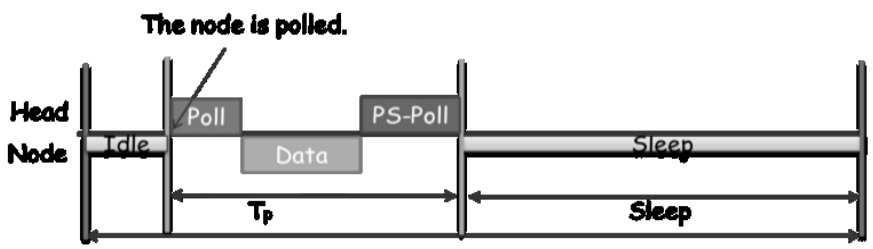

Fig. 5. Node processing.

\section{PERFORMANCE ANALYSIS}

\section{A. Energy Analysis}

We first calculate the time a cluster head spends to execute various states in a round, and then calculation the overall energy consumption by the energy consumption of each state.

TABLE I. VARIABLES OF THE ENERGY CONSUMPTION FORMULAS

\begin{tabular}{|l|l|}
\hline \multicolumn{2}{|c|}{ Variable } \\
\hline $\boldsymbol{T}$ & Round time $=1 / r$ \\
\hline $\boldsymbol{r}$ & Sample rate \\
\hline $\boldsymbol{T}_{\boldsymbol{p}}$ & Time for node processing \\
\hline $\boldsymbol{K}$ & Number of nodes \\
\hline $\boldsymbol{L}_{\boldsymbol{p}}$ & Length of a packet \\
\hline $\boldsymbol{C}_{\boldsymbol{r}}$ & Channel rate \\
\hline
\end{tabular}

According to the aforementioned works, we can deduce that the share of each state in each round. The variables refer to Table I.

Sleeping time is the time of each round without taking in operation:

$$
T_{s}=T-T_{p} \times K
$$

Where $\mathrm{T}_{\mathrm{p}}=$ Polling time + PS_POLL time $+\mathrm{T}_{\mathrm{r}}$ 
Transmitting time, Polling and PS-Poll are parts of the transmission, but also must be considered on each round how many times received:

$$
T_{t}=\text { Polling time }+ \text { PS_POLL time }
$$

Receiving time is the relationship of the size of the data bandwidth:

$$
T_{r}=\frac{L_{p}}{C_{r}}
$$

Energy calculation section we refer to the actual measurement of the resulting data to calculate the resulting energy consumption in this paper:

$$
\begin{array}{lc}
\text { Sending power } & P_{t}=280 \mathrm{~mA} \\
\text { Receiving power } & P_{r}=204 \mathrm{~mA} \\
\text { Sleeping power } & P_{S}=14 \mathrm{~mA}
\end{array}
$$

According to the time and energy above, we can deduce the overall energy consumption, and the results are as follows:

Energy consumption in a round is:

$$
E=T_{s} \times P_{S}+\left(T_{r} \times P_{r}+T_{t} \times P_{t}\right) \times K
$$

Substituting the result of (1), (2) and (3) into (4), the formula can be obtained by the following formula results:

$E=\frac{1}{r} \times P_{S}+\left(T_{r} \times P_{r}+\left(\right.\right.$ Polling $\left.+P S_{\text {POLL }}\right) \times P_{t^{-}}($Polling + $\left.\left.P S_{-} P O L L+T_{r}\right) \times P_{S}\right) \times K$

Consequently, the total energy consumption calculation formula of the cluster head is as follows:

Total energy consumption $=E \times$ total time $\times$ time slot

\section{Simulation Result}

The simulation environment is refer to [15] and [16], In this part, we compare the proposed scheme and send-then-forget mechanism, for these two models, we will separate their energy analysis, success rate analysis, delay time analysis and system life-time analysis, objective comparison of the pros and cons of the three systems.

TABLE II. THE PARAMETERS OF SIMULATION

\begin{tabular}{|cc|}
\hline \multicolumn{2}{|c|}{ Parameter } \\
\hline Sample period & 30000 unit time \\
\hline Polling time & 5 unit time \\
\hline Polling time & 5 unit time \\
\hline PS-Poll time & 1 unit time \\
\hline Data time & 50 unit time \\
\hline Tolerable time of nodes & 23017 unit time \\
\hline Energy consumption of sending & $280 \mathrm{~mA}$ \\
\hline Energy consumption of receiving & $204 \mathrm{~mA}$ \\
\hline Energy consumption of sleeping & $14 \mathrm{~mA}$ \\
\hline
\end{tabular}

\begin{tabular}{|cc|}
\hline Head power capacity & $1.31072 * 10^{11} \mathrm{mAh}$ \\
\hline Channel rate & $2 \mathrm{Mbps}$ \\
\hline
\end{tabular}

First, we define the basic environment. Our simulation environment is built in a circle, featuring the cluster head as the center, with a radius of 250 units. The coordinates $(100,100)$ are assumed to be the location of the cluster head, with uniform and random distributions of the 581 sensor nodes are in this region. Other parameters are listed in Table II.

In Fig. 6, the vertical axis represents the lifetime of the cluster head, and the horizontal axis represents the number of cluster nodes. We consider that the energy consumption in our method will be dynamically increased with the increasing of the node. In this way, we can reduce unnecessary waste by according to the amount of nodes. This effect is because we have introduced the "sleeping" mechanism. When there is no node needing to transmit, the head will also enter sleeping mode to conserve battery power. In addition, our approach will no longer receive data in the time of the upper bound of nodes in the system. The reason is that the information are time out, even if it receives is for naught, that is, to reduce energy consumption by avoiding unnecessary receiving. Because of our sleeping mechanism and the filter mechanism in the initial step, the power saving is achieved, and the lifetime of the cluster head is also be prolonged.

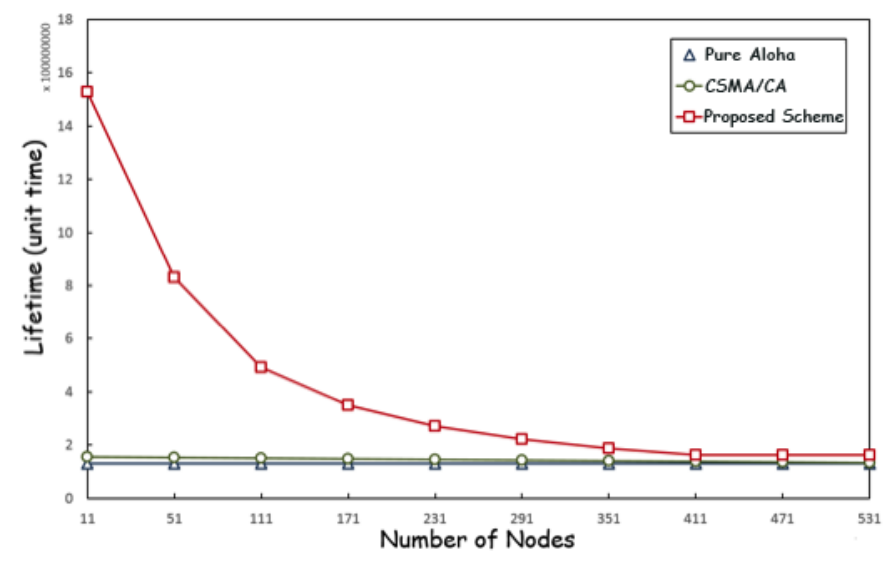

Fig. 6. Simulation of lifetime.

Fig. 7 shows the simulation of delay, the vertical axis represents the delay time of the cluster, and the horizontal axis represents the number of cluster nodes. From the figure, we can see that the delay time of the proposed scheme is similar to CS/MACA, but to the overall trend, the proposed scheme is better. In other words, our method has lower delay time. Because the data transmitting and receiving in cluster in our method will follow the schedule by using our "polling" method to make the cluster head have an absolutely effective data receiving. 


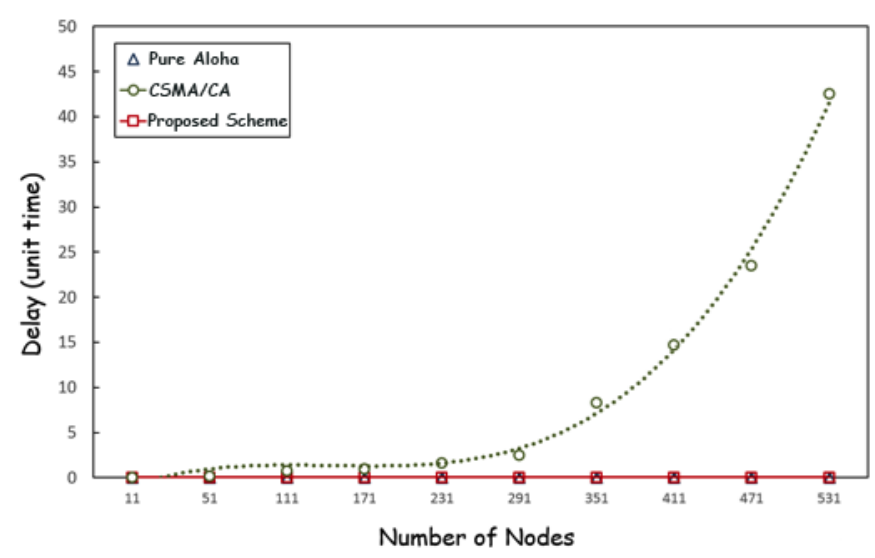

Fig. 7. Simulation of delay.

VI. CONCLUSION

Frequently data receiving and transmission will result in a sharp increase in energy consumption of cluster heads, and cause the opportunities that transform a cluster head into an energy hole substantial increase. Therefore, reducing the energy consumption of the cluster heads is an important key point. The focus of this paper is to find a way to save energy by scheduling the new ways to operate so that data can be transferred more quickly and correctly to the. The proposed method is based on a pragmatic polling with no collision priority-based access control scheme built on well-known protocols. The contributions of this paper include the following: First, through the conditions of organizing a cluster, the process of data receiving and transmission will successfully work without any connection timeout problem. The "polling" to make the cluster head has an absolutely effective data reception. Additionally, the "sleeping" mechanism ensures that the cluster can provide the most effective data reception under the premise of saving more energy for the cluster head. The most important goal is to extend the lifetime of the cluster head, and prioritize the optimization of the overall performance of the cluster. Through rigorous experiments and simulations, we contend both theoretically and experimentally that the proposed method has demonstrated a satisfactory performance.

\section{REFERENCES}

[1] J. Yick, B. Mukherjee, and D. Ghosal, "Wireless sensor network survey," Computer. Networks, vol. 52, no. 12, pp. 2292-2330. April 2008.

[2] H. Jiang, S. Jin, and C. Wang, "Prediction or not? An energy-efficient framework for clustering-based data collection in wireless sensor networks," IEEE Transactions on Parallel and Distributed Systems, vol. 22, no. 6, pp. 1064-1071, 2011.

[3] S. D. Muruganathan, D. C. F. Ma, R. I. Bhasin, and A. O. Fapojuwo,"A centralized energy-efficient routing protocol for wireless sensor networks," IEEE Commun. Mag., vol. 43, pp. S8-S13, Mar. 2005.

[4] M. Noori and M. Ardakani, "Characterizing the traffic distribution in linear wireless sensor networks," IEEE Commum. Lett., vol. 12, no. 8,pp. 554-556, Aug. 2008.

[5] Dietrich and F. Dressler, "On the lifetime of wireless sensor networks," ACM Transactions on Sensor Networks, vol. 5, no. 1, article 5, 2009.

[6] J. Li and P. Mohapatra, "An analytical model for the energy hole problem in many-to-one sensor networks," Proc. IEEE 62nd Vehicular Technology Conf., vol. 4, pp. 2721- 2725, Sept. 2005.

[7] M. Hajiaghayi, M. Dong, and B. Liang, "Maximizing lifetime in relay cooperation through energy-aware power allocation," IEEE Transactions on Signal Processing, vol. 58, no. 8, pp. 4354-4366, 2010.

[8] C. C. Lin, H. H. Chin, and D. J. Deng,"Dynamic Multi-Service Load Balancing in Cloud-based Multimedia System," IEEE System Journal, Vol. 8, Issue 1, pp. 225-234, 2014.

[9] J. Li and P. Mohapatra, "Analytical modeling and mitigation techniques for the energy hole problem in sensor networks," Pervasive Mob. Compiit., vol. 3, pp. 233-254, Jun. 2007.

[10] M. Noori and M. Ardakani, "Characterizing the traffic distribution in linear wireless sensor networks," IEEE Comimm. Lett., vol. 12, no. 8, pp. 554-556, Aug. 2008.

[11] D. J. Deng, C. H. Ke, H. H. Chen, and Y. M. Huang, "Contention Window Optimization for IEEE 802.11 DCF Access Control," IEEE Transactions on Wireless Communications, Vol. 7, No. 12, pp. 5129-5135, 2008.

[12] D. J. Deng and H. C. Yen, "Quality-of-Service Provisioning System for Multimedia Transmission in IEEE 802.11 Wireless LANs," IEEE Journal on Selected Areas in Communications, Vol. 23, No. 6, pp. 1240-1252, 2005.

[13] D. J. Deng and R. S. Chang, "A Non-preemptive Priority Based Access Control Scheme for Broadband Ad-Hoc Wireless ATM Local Area Networks," IEEE Journal on Selected Areas in Communications, Vol. 18, No. 9, pp. 1731-1739, 2000.

[14] D. J. Deng and R. S. Chang, "A Priority Scheme for IEEE 802.11 DCF Access Method," IEICE Trans. Commun., Vol. E82-B, No. 1, pp. 96-102, 1999.

[15] Benjie Chen, Kyle Jamieson, HariBalakrishnan and Robert Morris, "Span: An Energy-Efficient Coordination Algorithm for Topology Maintenance in Ad Hoc Wireless Networks," Wireless Networks, vol 8(5), Sep 2002, pp. 481-494.

[16] F.Bouabdallah,N.Bouabdallah,R.Boutaba,On Balancing Energy Consumption in Wireless Sensor Networks", IEEE Transactions on Vehicular Technology, vol.58, 2009. 